Utah State University

DigitalCommons@USU

7-10-2014

\title{
A New Guard-Band Call Admission Control Policy Based on Acceptance Factor for Wireless Cellular Networks
}

\author{
Asadur Rahman \\ Khulna University of Engineering \& Technology (KUET) \\ Mohammad Arif Hossain \\ Kookmin University \\ Shakil Ahmed \\ Utah State University, shakil.ahmed@aggiemail.usu.edu \\ Mostafa Zaman Chowdhury \\ Khulna University of Engineering \& Technology (KUET)
}

Follow this and additional works at: https://digitalcommons.usu.edu/ece_stures

Part of the Electrical and Computer Engineering Commons

\section{Recommended Citation \\ Md. Asadur Rahman, Mohammad Arif Hossain, Shakil Ahmed, and Mostafa Zaman Chowdhury, "A New Guard-Band Call Admission Control Policy Based on Acceptance Factor for Wireless Cellular Networks," In Proceeding 3rd IEEE International Conference on Informatics, Electronics and Vision (ICIEV),23-24 May 2014, pp. 1-5.}

This Conference Paper is brought to you for free and open access by the Electrical and Computer Engineering Student Works at DigitalCommons@USU. It has been accepted for inclusion in Electrical and Computer Engineering Student Research by an authorized administrator of DigitalCommons@USU. For more information, please contact digitalcommons@usu.edu.

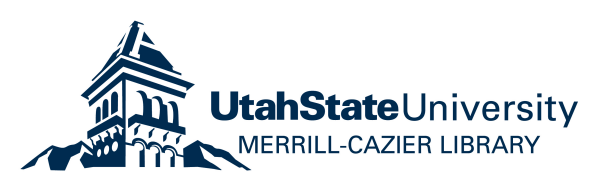




\title{
A New Guard-Band Call Admission Control Policy Based on Acceptance Factor for Wireless Cellular Networks
}

\author{
Md. Asadur Rahman, Mohammad Arif Hossain, Shakil Ahmed, and Mostafa Zaman Chowdhury \\ Department of Electrical and Electronic Engineering \\ Khulna University of Engineering \& Technology, Khulna-9203, Bangladesh \\ E-mail: eeeasadur@yahoo.com,dihan.kuet@gmail.com, shakileee076@gmail.com,mzceee@yahoo.com
}

\begin{abstract}
Utilization of limited resources and quality of service (QoS) improvement are the major concerns for wireless communication networks. Excessive call blocking is a constraint to attain the desired QoS. In cellular network, as the traffic arrival rate increases, new call blocking probability (CBP) increases considerably. Paying profound concern, we have proposed a guard-band call admission control policy that reduces the new call blocking probability with approximately steady handover call dropping probability (CDP) that ensures QoS. Our proposed scheme introduces the acceptance factor in specific guard channels where new calls get access according to the acceptance factor. The analytical results prove better performances than the conventional new-call bounding scheme and fractional guard channel (FGC) policy.
\end{abstract}

Keywords-Call admission control (CAC), call blocking probability (CBP), quality of service $(Q o S)$, acceptance factor, call dropping probability (CDP), new-call bounding scheme.

\section{INTRODUCTION}

The cellular communication is one of the best techniques in wireless communication systems. Since the demand for the cellular network has increased, there has been tremendous interest and progress in the field of wireless communications [1]. The physical area covered by this cellular network is divided into different specific regions called cells. When a mobile user crosses the cell boundary or the quality of the wireless link is unacceptable, then the process of handover call is initiated [2]. In recent years, a remarkable tendency in designing the wireless cellular network is- (i) decreasing the cell size and (ii) increasing the user mobility. These two factors result in more frequent handovers in wireless communication systems [3].

A call admission control (CAC) scheme aims to maintain the delivered quality of service (QoS) to the different calls at the target level by limiting the number of enduring calls in the system. One major challenge in designing a CAC arises to provide service two major types of calls: new or originating calls and handover calls. The QoS performances related to these two types of calls are generally measured by new call blocking probability and handover call dropping probability. In general, users are more sensitive to dropping of an ongoing and handed over call than blocking a new call [4].
Every CAC scheme has certain constraint to reduce the network blockage and termination of new calls and handover calls. Since blocking a new call is less serious than dropping a handover call, CAC schemes usually give a higher priority to handover calls [5]. In [1]-[9], some CAC schemes have been proposed. In these CAC schemes, there is a tradeoff between handover calls and new calls. That means due to provide priority to the handoff calls, the blocking probability of new calls increases.

In this paper, we propose a new guard-band CAC scheme based on new-call bounding scheme [7] and acceptance probability of call arrival rate. A new call can be accepted by a predefined probability on the basis of the present value of the state. This type of idea is proposed in [1]. Besides this acceptance factor can be varied depending on the call arrival rate which is proposed in [7]. In our proposed scheme we choose a specific value of acceptance factor throughout the band.

By this scheme we have shown that in a specific new-call bounding scheme blocking probability can be reduced significantly without changing the handover call dropping probability. The novelty of the acceptance factor is to determine the lower new call blocking probability for lower and higher call arrival rate. Furthermore, we compare our CAC scheme with fractional guard channel (FGC) scheme [1], [9] which proves better performance in the aspect of QoS. We also describe the impact of different values of acceptance factor on new call blocking probability.

This paper is organized as follows: Section II shows the hypothesis on handover and new call ratio. The new-call bounding scheme is explained in Section III. In Section IV, we represent our proposed guard-band CAC scheme. The performances of this paper are analyzed in Section V. Finally conclusions about the total work are drawn in Section VI.

\section{HYPOTHESIS ON HANDOVER \& NEW CALL RATIO}

In cellular networks, the rate of new call and handover call does not maintain the fixed ratio. This is why a hypothesis is necessary to obtain the relation between them. The relation among the originating or new call arrival rate $\left(\lambda_{n}\right)$, the handover call arrival rate $\left(\lambda_{h}\right)$, and the average channel departure rate $(\mu)$ 
is essential to determine the probability of blocking the new call and dropping the handover call. Here, it is considered that $P_{B}$ and $P_{D}$ represent the blocking probability of new calls and the dropping probability of handover calls, respectively. All calls arriving processes are assumed to be as Poisson's distributed.

A new call that arrives in the system may be either completed within the original cell or handed over to another cell before completing the call. The probability of handover of a call depends on two factors, (i) the average dwell time $(1 / \eta)$ and (ii) the average call duration $\left(1 / \mu_{a}\right)$ [4]. Again the average channel departure rate $(\mu)$ also depends on the above two parameters. Since both the call duration and the cell dwell time are assumed to be exponential, the handover probability, $P_{h}$ of a call at a particular time is given by [11],

$$
P_{h}=\frac{\eta}{\eta+\mu_{a}}
$$

and the handover call arrival rate into a cell is evaluated as,

$$
\lambda_{h}=\frac{\left(1-P_{B}\right) P_{h}}{\left[1-P_{h}\left(1-P_{D}\right)\right]}
$$

where the equation agrees from balancing the rates of handover calls into and out of a cell.

When a call is originated in a cell and gets a channel, the call holds the channel until the call is completed in the cell or the mobile moves out of the cell. Therefore, the channel holding time $T_{C}$ is either dwell time, $T_{h}$ or the call length time, $T_{n}$ [11]. Then the relation among them can be represented as following below,

$$
T_{c}=\min \left(T_{h}, T_{n}\right)
$$

\section{NEW-CALl Bounding SCHEME}

New-call bounding scheme is a general priority scheme. In this case, priority is given to handover requests by assigning guard channels $\left(G_{C}\right)$ entirely for handover calls among the $C$ channels in a cell. The rest $M\left(=C-G_{C}\right)$ channels are shared by both new calls and handover calls [10]. A new call is blocked if the progressive call is in state $M$ or more than that. A handover call is blocked if no channel is accessible in the target cell that means the operating state is at $C$.

The state $i(i=0,1 \ldots C)$ of a cell is defined as the number of calls in progress for the base station (BS) of that cell. Let $P(i)$ be the steady-state probability that the BS is in state $i$. The probabilities $P(i)$ can be found by analyzing the typical way of birth-death processes of one dimensional Markov chain [11]. The relevant state transition rate diagram is shown in Figure 1. This figure clarifies the birth rate of new and handover calls as well as the death rate of them by average departure rate $(\mu)$. From this figure, the state balance equations can be represented as,

$$
i \mu P(i)= \begin{cases}\left(\lambda_{n}+\lambda_{h}\right) P(i-1) & 0 \leq i \leq M \\ \lambda_{h} P(i-1) & M<i \leq C\end{cases}
$$

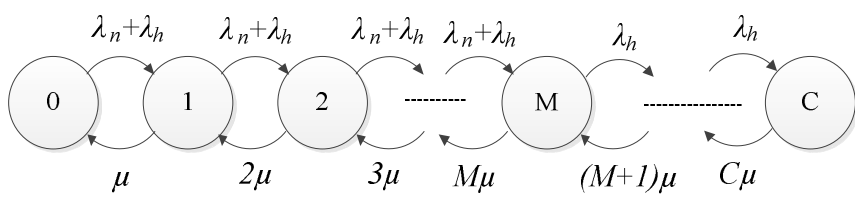

Figure 1: State transition rate diagram of new-call bounding scheme.

The steady-state probability $P(i)$ is found as follows:

$$
P(i)= \begin{cases}\frac{\left(\lambda_{n}+\lambda_{h}\right)^{i}}{i ! \mu^{i}} P(0) & 0 \leq i \leq M \\ \frac{\left(\lambda_{n}+\lambda_{h}\right)^{M} \lambda_{h}^{i-M}}{i ! \mu^{i}} P(0) & M<i \leq C\end{cases}
$$

where

$$
P(0)=\left[\sum_{i=0}^{M} \frac{\left(\lambda_{n}+\lambda_{h}\right)^{i}}{i ! \mu !}+\sum_{i=M+1}^{C} \frac{\left(\lambda_{n}+\lambda_{h}\right)^{M} \lambda_{h}{ }^{i-M}}{i ! \mu !}\right]^{-1}
$$

The blocking probability, $P_{B}$ for a new call is given by,

$$
P_{B}=\sum_{i=M}^{C} P(i)
$$

By this way, the blocking probability of handover request or dropping probability, $P_{D}$ is given by,

$$
P_{D}=\frac{\left(\lambda_{n}+\lambda_{h}\right)^{M} \lambda_{h}^{C-M}}{C ! \mu^{C}} P(0)=P(C)
$$

\section{PROPOSED CAC SCHEME}

In the proposed scheme, we use the basic idea of new-call bounding scheme and also a special guard band inside the channels that accepts the new calls with a defined acceptance factor and rejects the rest new calls. This guard band is assigned between priority and non-priority bands by taking some channels of the guard band from only handover accessing channels. This is why, in our proposed scheme, priority is given to the handover call by two steps. The state transition rate diagram of the system is described clearly by Markov chain in Figure 2. The three steps of the total channel $(C)$ allocation can be categorized briefly as following below.

1. O-M channels can be used by handover and new calls with same acceptance probability

2. M-N channels are allocated for handover and new calls with specific acceptance probability. Though the new call is accepted with the factor $\alpha$, the handoff call will be accepted with the probability 1.

3. Rest $\mathrm{N}-\mathrm{C}$ channels are allocated for handover calls only. Here, new call will be blocked totally.

The proposed scheme is designed mathematically in such a way that when the value of acceptance probability of this scheme becomes zero, this scheme turns to be same as new-call 


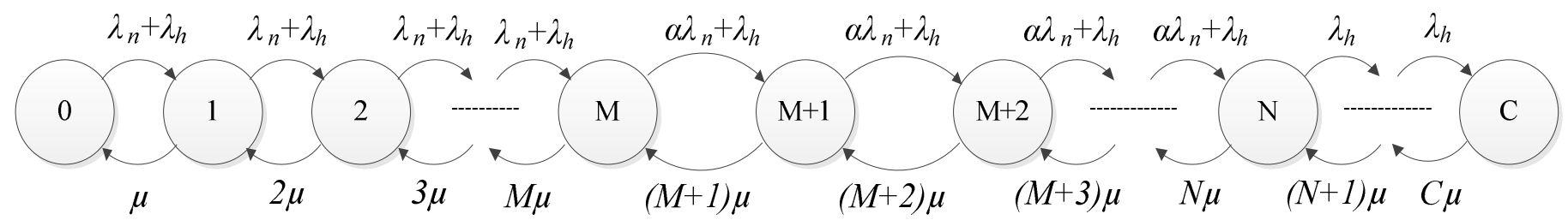

Figure 2: State transition rate diagram of the proposed scheme.

bounding scheme. Besides, the value of acceptance factor 1 shows the characteristics of non-priority scheme. Since the acceptance factor is chosen as one specific value throughout the band that reduces the computational complexity than the FGC scheme. If the new call is accepted as the probability of $\alpha$, the blocking probability will be $(1-\alpha)$. Depending on these ideas, the mathematical expressions are set as following.

The steady-state probability $P(i)$ is easily found as follows:

$$
P(i)= \begin{cases}\frac{\left(\lambda_{n}+\lambda_{h}\right)^{i}}{i ! \mu^{i}} P(0) & 0 \leq \mathrm{i} \leq \mathrm{M} \\ \frac{\left(\lambda_{n}+\lambda_{h}\right)^{M}\left[\alpha \lambda_{n}+\lambda_{h}\right]^{i-M}}{i ! \mu^{i}} P(0) & \mathrm{M}<\mathrm{i} \leq \mathrm{N} \\ \frac{\left(\lambda_{n}+\lambda_{h}\right)^{M}\left[\alpha \lambda_{n}+\lambda_{h}\right]^{M-N} \lambda_{h}^{i-N}}{i ! \mu^{i}} P(0) & \mathrm{N}<\mathrm{i} \leq \mathrm{C}\end{cases}
$$

where,

$$
\begin{aligned}
P(0)= & {\left[\sum_{i=0}^{M} \frac{\left(\lambda_{n}+\lambda_{h}\right)^{i}}{i ! \mu^{i}}+\sum_{i=M+1}^{N} \frac{\left(\lambda_{n}+\lambda_{h}\right)^{M}\left[\alpha \lambda_{n}+\lambda_{h}\right]^{i-M}}{i ! \mu^{i}}+\right.} \\
& \left.\sum_{i=N+1}^{C} \frac{\left(\lambda_{n}+\lambda_{h}\right)^{M}\left[\alpha \lambda_{n}+\lambda_{h}\right]^{N-M} \lambda_{h}^{i-N}}{i ! \mu^{i}}\right]^{-1}
\end{aligned}
$$

The blocking probability $P_{B}$ for a new call according to (9)(10) is given by,

$$
\begin{gathered}
P_{B}=\frac{\left(\lambda_{n}+\lambda_{h}\right)^{M}(1-\alpha)\left(\alpha \lambda_{n}+\lambda_{h}\right) P(0)}{\mu^{M+1}} \times \\
\sum_{i=1}^{N-M} \frac{\left[\alpha \lambda_{n}+\lambda_{h}\right]^{i-1}}{(M+1) ! \mu^{i}}+P(0) \sum_{i=N+1}^{C} P(i)
\end{gathered}
$$

The dropping probability for handover request will be same as the new-call bounding scheme that means when the ongoing call is in the state $C$. Hence the probability of having the request of handover call at the state $C$ is the handover call dropping probability, $P_{D}$ which is given by,

$$
P_{D}=P(C)
$$

From (9)-(11), it is clear that our proposed guard-band CAC scheme is convertible to the new-call bounding scheme depending on the value of acceptance factor as we have mentioned earlier. According to the value of acceptance factor the blocking probability fluctuates in nonlinear pattern about which we have explained in the next section.

\section{PERFORMANCE ANALYSIS}

The proposed scheme and the conventional new-call bounding scheme are analyzed considering average call life time, $1 / \mu_{a}=120$ second and average cell dwell time, $1 / \eta=360$ second. Total number of channels, $C$ in both cases is taken as 120.

Handover and new call equally sharing channel number, $M$ is taken 100. According to the proposed scheme the special guard-band with acceptance factor is defined by 10 channels which is allocated between $M$ and $N$, this is why $N=110$.

In our proposed scheme, it is necessary to find the value of acceptance factor that shows the minimum new call blocking and handover call dropping probability. In this case, we have analyzed the value of acceptance factor by iterative method from 0.1 to 0.9 . We have found that the value of acceptance factor $(\alpha)$ that demonstrates minimum blocking probability and steady dropping probability throughout the call arrival rates is 0.9 .

By this consideration, taking $\alpha=0.9$ the blocking probability of new calls and the dropping probability of handover calls for the proposed scheme and these probability for new-call bounding scheme have been shown in Figure 3. In this figure, we observe that our proposed scheme shows the lower new call blocking probability keeping the dropping probability considerably fixed. Moreover, we compare our performances with FGC scheme in Figure 4. The figure depicts that the call blocking and dropping probability of our proposed scheme maintain better QoS than the FGC scheme.

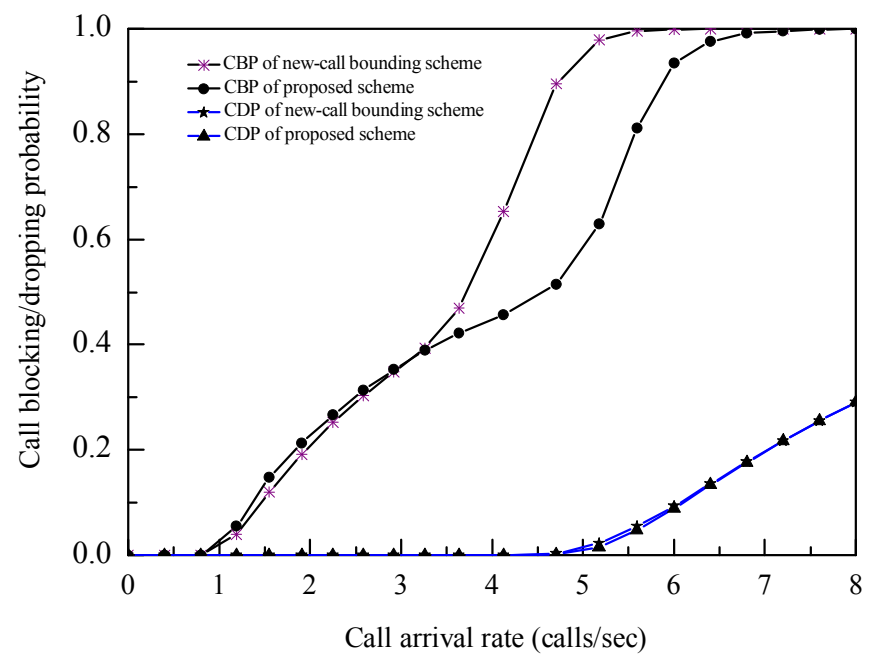

Figure 3: Comparison of call blocking and call dropping probability between new-call bounding scheme and proposed scheme. 


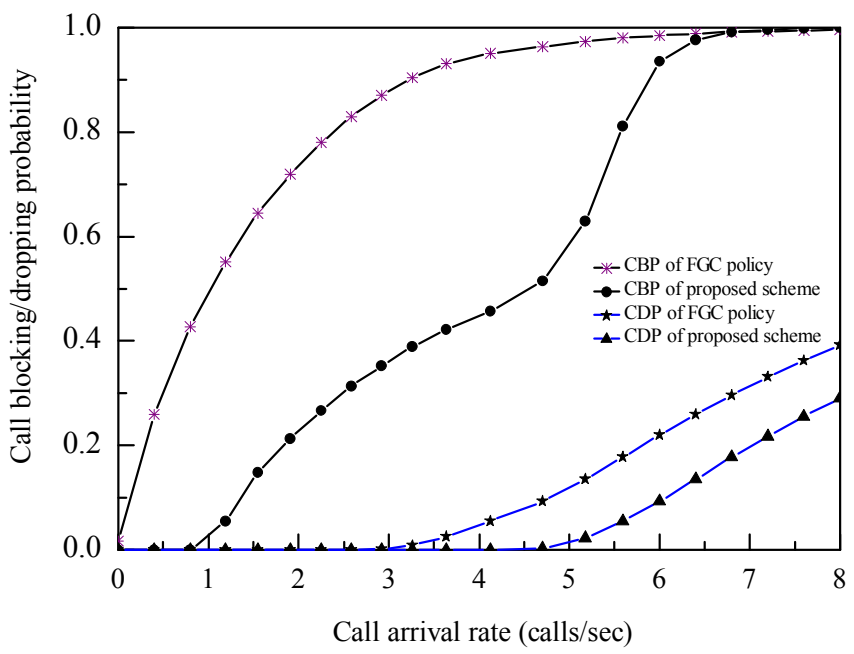

Figure 4: Comparison of call blocking and dropping probability between fractional guard channel (FGC) scheme and proposed scheme

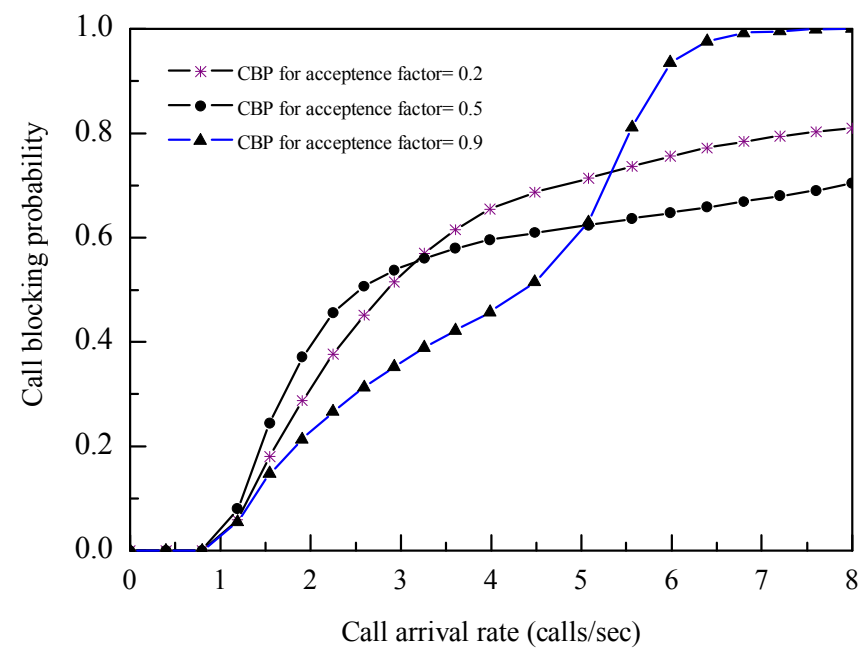

Figure 5: Comparison of call blocking probability with different acceptance factor.

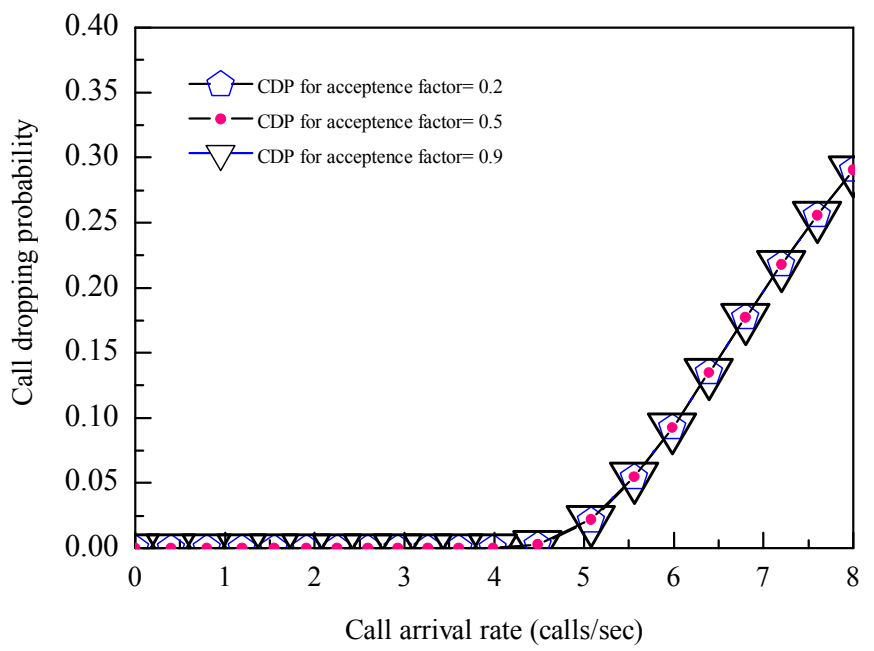

Figure 6: Comparison of call dropping probability with different acceptance factor.

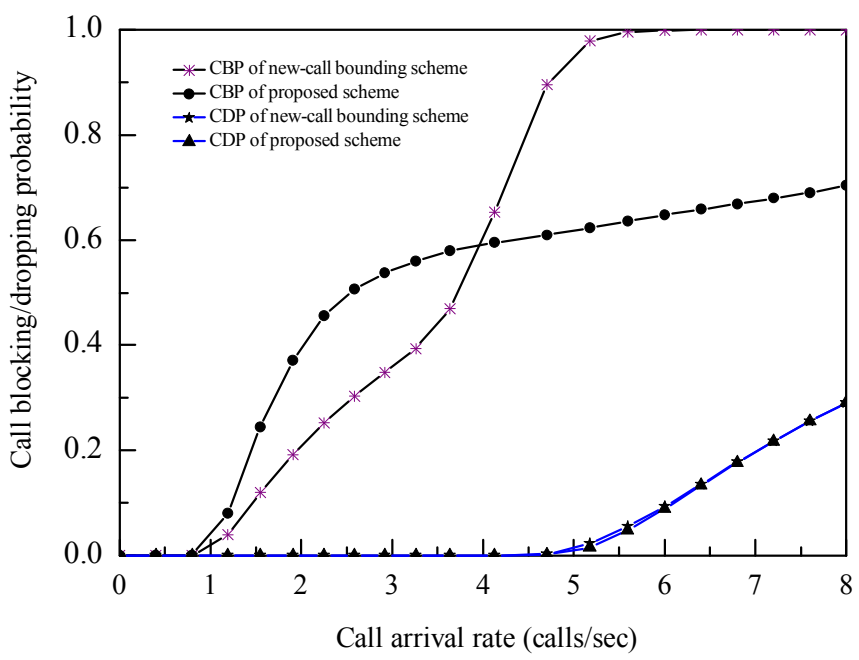

Figure 7: Comparison of proposed scheme and new-call bounding scheme for acceptance ratio of 0.5 .

In Figure 5, we present a comparison among the new call blocking probability for different acceptance factor. Form the figure it has been observed that at higher traffic rate the call blocking probability of acceptance factor 0.5 shows lower than the other. When the traffic rate is lower, 0.5 shows the poorest performance. Therefore, we choose the acceptance factor 0.5 only when a big burst of new calls originated in BS. In the case of lower traffic rate the acceptance factor 0.9 shows the minimum blocking probability than the other. As we assured, in every case the dropping probability of handover calls are considerably constant which has been shown in Figure 6 .

In Figure 7, we present a comparison of new call blocking probability and handover call dropping probability between new-call bounding scheme and our proposed scheme with acceptance factor 0.5 . As we mentioned, the value of acceptance factor 0.5 shows the lowest blocking probability at the very higher traffic load. This criterion cannot be chosen for the lower call arrival rates due to its higher new call blocking probability.

\section{CONCLUSIONS}

In this paper, an efficient guard-band CAC scheme has been proposed which combines the idea of new-call bounding scheme and call acceptance dependent CAC scheme. From the derived equations of the proposed scheme we can return back to new-call bounding CAC scheme. This proposed CAC scheme ensures a minimum permissible new call blocking probability keeping the handover call dropping probability almost constant as new-call bounding scheme. Our proposed scheme contains two guard-bands. One of these two bands accepts the new calls with a given acceptance factor that ensures better performance. Else, our proposed scheme shows much better performance than FGC policy in the aspect of QoS. This work clarifies to choose the best value of acceptance factor whether the traffic arrival rate is lower or higher. In our future work, we will research on CAC policy using two dimensional Markov chain for multiclass traffic. 


\section{REFERENCES}

[1] R. Ramajee, R. Nagarajan, and D. Towsley, "On optimal call admission control in cellular networks," Wireless Networks, vol. 3, no. 1, pp. 2941, March 1997.

[2] A. Sgora, Vergados, and Dimitrios D., "Handoff prioritization and decision schemes in wireless cellular networks: a survey," IEEE Communications Surveys and Tutorials, vol. 11, no. 4, pp. 57-77, December 2009.

[3] M. Z. Chowdhury, Y. Min Jang, and Z. J. Haas, "Call admission control based on adaptive bandwidth allocation forwireless networks," Journal of Communications and Networks, vol. 15, no. 1, pp. 15-24, February 2013.

[4] A. Leelavathi and G. V. Sridhar, "Adaptive bandwidth allocation in wireless networks with multiple degradable quality of service," IOSR Journal of Electronics and Communication Engineering, vol. 2, no. 4 , pp. 25-29, October 2012.

[5] M. Z. Chowdhury, M. S. Uddin, and Y. M. Jang, "Dynamic channel allocation for class-based QoS provisioning and call admission in visible light communication," Arabian Journal for Science and Engineering, vol. 39, no. 2, pp. 1007-1016, February 2014.
[6] Y. Fang, "Thinning scheme for call admission control in wireless networks," IEEE Transactions on Computers, vol. 52, no. 5, pp. 685687, May 2003.

[7] Y. Fang and Y. Zhang, "Call admission control schemes and performance analysis in wireless mobile networks," IEEE Transactions on Vehicular Technology, vol. 51, no. 2, pp. 371-382, March 2002.

[8] D. Hong and S. S. Rappaport, "Traffic model and performance analysis for cellular mobile radio telephone systems with prioritized and noprioritized handoff procedures," IEEE Transactions on Vehicular Technology, vol. 35, no. 3, pp. 77-92, August 1986.

[9] J. L. Vazquez Avila, F. A. Cruz Perez, and L. O. Guerrero, "Performance analysis of fractional guard channel policies in mobile cellular networks," IEEE Transactions on Wireless Communications, vol. 5, no. 2, pp. 301- 305, February 2006.

[10] Q. An Zeng and D. P. Agrawal, Handoff in wireless mobile networks, Department of Electrical Engineering and Computer Science, University of Cincinnati, by John Wiley \& Sons, Inc, 2002.

[11] M. Schwartz, Mobile wireless communications, Cambridge University Press, 2005. 